September, 1994

\title{
Symmetries of the Kac-Peterson Modular Matrices of Affine Algebras
}

\author{
Terry Gannon ${ }^{1}$ \\ Institut des Hautes Etudes Scientifiques \\ 91440 Bures-sur-Yvette, France
}

\section{$0 \quad$ Introduction}

One of the more fruitful observations in recent years was the discovery [19] that the (normalized) character $\chi_{\mu}$ of the integrable highest weight $g$-module $L(\mu)$ of a nontwisted Kac-Moody algebra $g=X_{r}^{(1)}$ is a modular form. Thanks to the structure of the affine Weyl group, these $\chi_{\mu}$ can be written as ratios of theta functions, so on them exists a natural action of $S L_{2}(\mathbf{Z})$. In fact, this action defines a representation

$$
R: S L_{2}(\mathbf{Z}) \rightarrow G L_{n}(\mathbf{C})
$$

on the space spanned by the $\chi_{\mu}$, for $\mu$ lying in the set $P_{+}(g, k)$ of all level $k$ highest weights of $g$ (the dimension $n$ equals the cardinality $\left\|P_{+}(g, k) \bmod \mathbf{C} \delta\right\|$, for the imaginary root $\delta$ of $g$ ). The matrices in the image $R\left(S L_{2}(\mathbf{Z})\right.$ ), which we will call the Kac-Peterson modular matrices, are unitary (in the $\chi_{\mu}$ basis).

This observation and its various consequences have found application in several areas. For example, many conformal field theories $[29,14,15]$ are intimately connected with nontwisted affine $g$. Much of their structure is encoded in their "genus 1 partition function", which in the case of a Wess-Zumino-Witten theory $[29,14]$ at level $k$ based on $g$ is a sesquilinear combination of characters of $g$ :

$$
Z=\sum_{\mu, \nu \in P_{+}(g, k) \bmod \mathbf{C} \delta} M_{\mu, \nu} \chi_{\mu} \chi_{\nu}^{*} .
$$

This function must satisfy some properties, namely:

- the coefficients $M_{\mu, \nu}$ in eq.(1) must be non-negative integers;

- $Z$ must be invariant under the action of $S L_{2}(\mathbf{Z})$ on the characters - equivalently, the matrix $M$ must commute with all matrices in $R\left(S L_{2}(\mathbf{Z})\right)$;

\footnotetext{
1 Present address: Math Dept, Concordia University, Montréal, Canada, H3G 1M8
} 
- $M_{k \Lambda_{0}, k \Lambda_{0}}=1$.

We shall call any such matrix $M$ a physical invariant. The classification of these physical invariants is a major problem in conformal field theory. Unfortunately, it is difficult and in spite of much effort little is known. What makes the problem more interesting though is that many startling coincidences have appeared between the few existing classifications and other areas of mathematics. For instance, the physical invariants corresponding to $g=A_{1}^{(1)}$ [5] fall into the A-D-E pattern familiar to e.g. singularity theory, the finite subgroups of $S_{2}(\mathbf{C})$, and of course the simply-laced Lie algebras. In the classification of the physical invariants for $g=A_{2}^{(1)}$ [10] a number of surprising relations [23,7] with the Jacobians of the Fermat curves [22] have been found. Recently [30], a more inclusive and sophisticated interpretation of some of these relationships has been proposed using generalized Coxeter graphs.

It should not be too surprising that as rich a subject as conformal field theory has subtle interconnections with other parts of mathematics. And indeed some have become established in recent years, thanks to the work of Witten, Kac, Verlinde, and many others. But the coincidences involving the CFT classifications are still poorly understood; it is difficult to know their significance or to anticipate how they extend to other affine algebras. For this reason, as well of course for the classification of conformal field theories itself, it is certainly of interest to try to find all physical invariants corresponding to e.g. $A_{r}^{(1)}$.

In Section 2 we classify an important subset of the $A_{r}^{(1)}$ physical invariants, called automorphism invariants (see eqs.(5)). These correspond to those $M$ in eq.(1) which are permutation matrices; equivalently, they are the exact symmetries of all the KacPeterson modular matrices of the affine algebra at a given level. We extend our proof to $A_{\vec{r}}^{(1)} \stackrel{\text { def }}{=}\left(A_{r_{1}} \oplus \cdots \oplus A_{r_{s}}\right)^{(1)}, \vec{r}=\left(r_{1}, \ldots, r_{s}\right)$ in Section 3 ; it should also extend to any other affine algebra, as well as a much larger class of physical invariants (namely all automorphisms of the Bernard [2] - i.e. simple current [25] - chiral extensions). The value of our result to the classification problem of CFT will be discussed in more depth at the end of Section 1.

A brief sketch of the proof is given at the beginning of Section 2. Among the tools we use in this paper are the Verlinde formula, and the fact that the Weyl character of any representation of a finite-dimensional complex simple Lie algebra $\bar{g}$ can be written as a polynomial in the Weyl characters of its fundamental representations. An important connection between the representation theory of $g$ and that of finite dimensional $\bar{g}$ is the fact (see eq.(3a)) that certain ratios of entries of the Kac-Peterson modular matrix $S$ equal certain values of the Weyl characters of $\bar{g}$. This connection is exploited throughout the paper.

In the remainder of this introduction we will briefly review the little that is known about physical invariants and their classification.

It is easy to show [9] that for any fixed algebra $g$ and level $k$, there will be only finitely many physical invariants. Many of these have already been constructed. Almost all known ones are built up from the symmetries Aut $\Pi^{\vee}$ of the extended Dynkin diagram, in simple ways $[2,1,25]$; they are called $\mathcal{D}$-type invariants (by analogy with the A-D-E classification of $A_{1}^{(1)}$ ). The remaining physical invariants are called exceptional (see e.g. [4,27]). Most 
known exceptionals are related to the so-called conformal subalgebras of $g[4,20]$ - i.e. subalgebras whose branching functions $b_{\mu}^{\lambda}(\tau)$ are constants.

The best known classification is the A-D-E one for $A_{1}^{(1)}$ [5]. There we find an $\mathcal{A}_{k^{-}}$ series (for all $k$ ) and $\mathcal{D}_{k}$-series (for even $k$ ) - both these are of $\mathcal{D}$-type. There are also three exceptionals, at levels 10, 16 and 28. The only other classifications known at present for the physical invariants of affine algebras $g$ are: $k=1$ when $\bar{g}$ is simple $[9,16] ; A_{2}^{(1)}$ for all $k$ [10]; and $\left(A_{1} \oplus A_{1}\right)^{(1)}$ for all $k=\left(k_{1}, k_{2}\right)[11]$.

We will prove that the only automorphism invariants for $A_{\vec{r}}^{(1)}$ are those of $\mathcal{D}$-type. Incidently, this is not the case for all affine algebras: e.g. there are exceptional automorphism invariants for $G_{2}^{(1)}$ at level 4 and $F_{4}^{(1)}$ at level 3 [27], and for infinitely many $B_{r}^{(1)}$ and $D_{r}^{(1)}$ at level 2 [12]. The methods developed here should apply to the other affine algebras [12], though the details will certainly be messier.

The only other existing major classification of automorphism invariants is for the "simple current automorphism invariants" [13] of any rational conformal field theory (subject to a condition on the corresponding modular matrix $S$ ). However the argument in [13] cannot apply here because it limits its attention to automorphism invariants of a special form not shared by most of the $A_{\vec{r}}^{(1)}$ automorphism invariants; it also assumes that (roughly speaking) the modular matrix $S$ in eq.(2a) does not have too many zeros - something known at present only for $A_{1}^{(1)}$ and $A_{2}^{(1)}$.

Years of effort with little accomplished has made most in the field skeptical about the possibility of a classification of all physical invariants, even for $A_{r}^{(1)}$. Our result, especially considering its simplicity, directly challenges this pessimism. Knowing all the automorphism invariants is a necessary and major step towards the full classification.

\section{The Kac-Peterson modular matrices}

In this section we will introduce some notation and terminology, and review some results in $[19,20]$. Our notation will remain as close as possible to [17]. We will focus here on $g=A_{r}^{(1)}$; analogous statements hold for the other affine algebras [17,21], and those for $A_{\vec{r}}^{(1)}$ will be given at the beginning of Section 3 .

Write $\bar{r}=r+1$ and $\bar{k}=k+\bar{r}$. Choose a Cartan subalgebra $h$ for $g$; its dual $h^{*}$ will be spanned by the fundamental weights $\Lambda_{0}, \ldots, \Lambda_{r}$ together with the imaginary root $\delta=\sum_{i=0}^{r} \alpha_{i}$. We can (p.155 of [17], eq.(1.5.12) of [20]) and will identify a highest weight

$$
\lambda=\sum_{i=0}^{r} \lambda_{i} \Lambda_{i}+z \delta \in h^{*}
$$

with its Dynkin labels $\left(\lambda_{0}, \lambda_{1}, \ldots, \lambda_{r}\right)$, and drop the $z$. In particular, the set of level $k$ highest weights for $A_{r}^{(1)}$ becomes

$$
P_{+}^{r, k} \stackrel{\text { def }}{=} P_{+}\left(A_{r}^{(1)}, k\right) \bmod \mathbf{C} \delta=\left\{\sum_{i=0}^{r} \lambda_{i} \Lambda_{i} \mid \lambda_{i} \in \mathbf{Z}, \quad \lambda_{i} \geq 0, \sum_{i=0}^{r} \lambda_{i}=k\right\}
$$


Put $\rho=\sum_{i=0}^{r} \Lambda_{i}$. The invariant bilinear form $(-\mid-)$ for the underlying finite-dimensional algebra $\bar{g}=A_{r}$, normalized so that the roots have norm 2, can be extended to $h^{*}$ by defining $\left(\Lambda_{0} \mid \Lambda_{i}\right)=\left(\Lambda_{i} \mid \Lambda_{0}\right)=(\delta \mid \delta)=0,\left(\delta \mid \Lambda_{i}\right)=\left(\Lambda_{i} \mid \delta\right)=\delta_{i, 0}, \forall i$. We will let $\bar{\lambda}$ denote the orthogonal projection $\left(\lambda_{1}, \ldots, \lambda_{r}\right)$ onto the dual $\bar{h}^{*}=\mathbf{C} \Lambda_{1} \oplus \cdots \oplus \mathbf{C} \Lambda_{r}$ of the Cartan subalgebra of $\bar{g}$. Let Aut $\Pi^{\vee}$ denote the group of automorphisms of the (extended) Dynkin diagram of $g$ : for $A_{r}^{(1)}$ it has generators $C_{r}$ and $J_{r}$ acting on $P_{+}^{r, k}$ by

$$
\begin{aligned}
C_{r} \lambda & =\lambda_{0} \Lambda_{0}+\sum_{i=1}^{r} \lambda_{\bar{r}-i} \Lambda_{i}, \\
J_{r} \lambda & =\lambda_{r} \Lambda_{0}+\sum_{i=1}^{r} \lambda_{i-1} \Lambda_{i} .
\end{aligned}
$$

Consider the irreducible integrable highest weight $g$-module $L(\lambda)$. Let $L(\lambda)=\oplus_{\beta} L(\lambda)_{\beta}$ denote its weight space decomposition with respect to $h$. Define the normalized character of $L(\lambda)$ to be

$$
\chi_{\lambda}=e\left(-m_{\lambda} \delta\right) \sum_{\beta} \operatorname{dim}\left[L(\lambda)_{\beta}\right] e(\beta),
$$

where $m_{\lambda}$ is a rational number (the modular anomaly), and for each $\gamma \in h^{*}, e(\gamma)$ can be taken to be the function $e(\gamma): h \rightarrow \mathbf{C}$ given by $e(\gamma)(v)=e^{\gamma(v)}$.

Coordinatizing $h$ appropriately [19], $\chi_{\lambda}$ may be regarded as a complex-valued function of $\bar{z} \in \bar{h}$ and complex variables $\tau, u$, such that for each $\lambda \in P_{+}^{r, k}$

$$
\begin{aligned}
& \chi_{\lambda}\left(\frac{-1}{\tau}, \frac{\bar{z}}{\tau}, u-\frac{(\bar{z} \mid \bar{z})}{2 \tau}\right)=\sum_{\mu \in P_{+}^{r, k}} S_{\lambda, \mu}^{r, k} \chi_{\mu}, \\
& S_{\lambda, \mu}^{r, k} \stackrel{\text { def }}{=} \frac{\exp [\pi i r \bar{r} / 4]}{\bar{k}^{r / 2} \sqrt{\bar{r}}} \sum_{w \in \bar{W}} \operatorname{det}(w) \exp \left[-2 \pi i \frac{(w(\bar{\lambda}+\bar{\rho}) \mid \bar{\mu}+\bar{\rho})}{\bar{k}}\right] ; \\
& \chi_{\lambda}(\tau+1, \bar{z}, u)=\sum_{\mu \in P_{+}^{r, k}} T_{\lambda, \mu}^{r, k} \chi_{\mu}, \\
& T_{\lambda, \mu}^{r, k} \stackrel{\text { def }}{=} \exp \left[\pi i \left\{\frac{(\lambda+\rho \mid \lambda+\rho)}{\bar{k}}-\frac{(\rho \mid \rho)}{\left.\left.h^{\vee}\right\}\right] \delta_{\lambda, \mu} .}\right.\right.
\end{aligned}
$$

We will often delete the superscripts ' $r, k$ ' in the following. $\bar{W}$ in eq.(2a) denotes the (finite) Weyl group of $A_{r}$. The transformations in eqs.(2) generate the complete action of $S L_{2}(\mathbf{Z})$ on the space spanned by the $\chi_{\lambda}$, and so these equations suffice to uniquely specify the representation $R$ of $S L_{2}(\mathbf{Z})$. The Kac-Peterson matrices $R\left(S L_{2}(\mathbf{Z})\right)$ consist of all possible products of $S^{r, k}$ and $T^{r, k}$.

The matrices $S^{r, k}$ and $T^{r, k}$ are unitary and symmetric. $\left(S^{r, k}\right)^{2}=C^{r, k}$, the matrix characterizing the action of $C_{r}$ on $P_{+}^{r, k}$. A remarkable property [19] of $S^{r, k}$, which we will use frequently, follows immediately from eq.(2a) and the Weyl character formula:

$$
\frac{S_{\lambda, \mu}}{S_{k \Lambda_{0}, \mu}}=c h_{\bar{\lambda}}\left(-2 \pi i \nu^{-1}(\bar{\mu}+\bar{\rho}) / \bar{k}\right) \text {, }
$$


where $\nu: \bar{h} \rightarrow \bar{h}^{*}$ is the isomorphism defined by $(-\mid-)$, and where $c h \bar{\lambda}$ denotes the Weyl character of the $A_{r}$-module $\bar{L}(\bar{\lambda})$ :

$$
c h_{\bar{\lambda}} \stackrel{\text { def }}{=} \sum_{\bar{\beta}} \operatorname{dim}\left[\bar{L}(\bar{\lambda})_{\bar{\beta}}\right] e(\bar{\beta}) .
$$

A special case of eq.(3a) is the $q$-dimension of any $\lambda \in P_{+}^{r, k}$ :

$$
Q^{r, k}(\lambda) \stackrel{\text { def }}{=} \frac{S_{\lambda, k \Lambda_{0}}}{S_{k \Lambda_{0}, k \Lambda_{0}}}=\prod_{\bar{\alpha}>0} \frac{\sin [\pi(\bar{\lambda}+\bar{\rho} \mid \bar{\alpha}) / \bar{k}]}{\sin [\pi(\bar{\rho} \mid \bar{\alpha}) / \bar{k}]}
$$

The $\bar{\alpha}>0$ in eq.(3b) are the positive roots of $A_{r}$. These considerations also imply [17]

$$
S_{k \Lambda_{0}, \lambda} \geq S_{k \Lambda_{0}, k \Lambda_{0}}>0, \quad \forall \lambda \in P_{+}^{r, k}
$$

Straightforward calculations give us [20]

$$
\begin{aligned}
t(\lambda) \stackrel{\text { def }}{=} & \bar{r}\left(\lambda \mid \Lambda_{r}\right)=\sum_{j=1}^{r} j \lambda_{j}, \\
t\left(J_{r}^{a} \lambda\right) & \equiv k a+t(\lambda) \quad(\bmod \bar{r}), \\
T_{J_{r}^{a} \lambda, J_{r}^{a} \mu}^{r, k} & =\exp [\pi i(-2 a t(\lambda)+k a(\bar{r}-a)) / \bar{r}] T_{\lambda, \mu}^{r, k}, \\
S_{J_{r}^{a} \lambda, J_{r}^{b} \mu}^{r, k} & =\exp [2 \pi i(b t(\lambda)+a t(\mu)+k a b) / \bar{r}] S_{\lambda, \mu}^{r, k}, \\
S_{C_{r} \lambda, \mu}^{r, k} & =S_{\lambda, C_{r} \mu}^{r, k}=S_{\lambda, \mu}^{r, k *}, \quad T_{C_{r} \lambda, C_{r} \mu}^{r, k}=T_{\lambda, \mu}^{r, k} .
\end{aligned}
$$

Incidently, there is a Galois symmetry $[9,23,6]$ obeyed by $S^{r, k}$ which has proven to be valuable in some contexts (see e.g. [10]). Although we will not use it here, it is sufficiently little known to warrant us repeating. We see from eq.(2a) that the entries of $S^{r, k}$ lie in the cyclotomic extension $\mathbf{Q}(\exp [\pi i / 2 \bar{k} \bar{r}])$ of the rationals $\mathbf{Q}$. Choose any element $g$ of the corresponding Galois group. Then associated to $g$ is a permutation $\lambda \mapsto \lambda^{g}$ of the weights in $P_{+}^{r, k}$, as well as a map $\epsilon_{g}: P_{+}^{r, k} \rightarrow\{ \pm 1\}$, such that

$$
g\left(S_{\lambda, \mu}\right)=\epsilon_{g}(\lambda) S_{\lambda^{g}, \mu}=\epsilon_{g}(\mu) S_{\lambda_{, \mu} g}, \quad \forall \lambda, \mu \in P_{+}^{r, k}
$$

The identical result holds for any other nontwisted affine algebra. This Galois symmetry reappears throughout rational conformal field theory: for instance the matrix $M$ in eq.(1) must obey

$$
M_{\lambda, \mu}=\epsilon_{g}(\lambda) \epsilon_{g}(\mu) M_{\lambda^{g}, \mu^{g}}, \quad \forall \lambda, \mu \in P_{+}(g, k) \bmod \mathbf{C} \delta .
$$

Definition. An automorphism invariant of $g$ at level $k$ is a permutation $\sigma$ of $P_{+}(g, k)$ mod $\mathbf{C} \delta$ such that $U_{\lambda, \mu}=U_{\sigma \lambda, \sigma \mu}$ for all Kac-Peterson matrices $U \in R\left(S L_{2}(\mathbf{Z})\right)$ and all $\lambda, \mu \in P_{+}(g, k) \bmod \mathbf{C} \delta$. 
Remarks. Since any such $U$ is generated by the analogues of the matrices $S$ and $T$ in eqs.(2), it suffices to require that $\sigma$ satisfy

$$
\begin{array}{ll}
S_{\lambda, \mu}=S_{\sigma \lambda, \sigma \mu}, & \forall \lambda, \mu \in P_{+}(g, k) \bmod \mathbf{C} \delta, \\
T_{\lambda, \mu}=T_{\sigma \lambda, \sigma \mu}, & \forall \lambda, \mu \in P_{+}(g, k) \bmod \mathbf{C} \delta .
\end{array}
$$

Our task in this paper is to find all automorphism invariants for $A_{\vec{r}}^{(1)}$. The simplest examples are $\sigma=C_{r_{1}}^{c_{1}} \otimes \cdots \otimes C_{r_{s}}^{c_{s}}$ for any choice of $c_{i} \in \mathbf{Z}$ - these obey eqs.(5) because of eq.(4e). Of course the set of all automorphism invariants will be a group under composition. Because $k \Lambda_{0}$ is the only row of $S$ which is strictly positive, we see that

$$
\sigma\left(k \Lambda_{0}\right)=k \Lambda_{0}
$$

Defining $M$ by $M_{\lambda, \mu}=\delta_{\mu, \sigma \lambda}$, we find that any automorphism invariant is a physical invariant. The converse is usually not true; nevertheless the automorphism invariants are an important subset of the physical invariants which until now were quite intractible. For one thing, the matrix product of an automorphism invariant with any other physical invariant will also be a physical invariant. Also, knowing the automorphism invariants for affine algebras automatically means we know all automorphisms of those exceptional chiral extensions due to conformal subalgebras $[4,20]$ - the main source of exceptional extensions. Finally, knowing the automorphism invariants should permit the classification of all physical invariants $M$ with the property:

$$
M_{\lambda, k \Lambda_{0}}, M_{k \Lambda_{0}, \mu} \neq 0 \Rightarrow \exists J, J^{\prime} \in A u t \Pi^{\vee} \text { such that } \lambda=J\left(k \Lambda_{0}\right), \mu=J^{\prime}\left(k \Lambda_{0}\right) .
$$

This would be important because almost every known physical invariant obeys that property. Of course, any automorphism invariant satisfies it - in fact a physical invariant $M$ will be an automorphism invariant iff $M_{\lambda, k \Lambda_{0}}=M_{k \Lambda_{0}, \lambda}=\delta_{\lambda, k \Lambda_{0}} \forall \lambda \in P_{+}(g, k) \bmod \mathbf{C} \delta$ [9].

\section{The automorphism invariants of $A_{r}^{(1)}$}

Our goal in this paper is to find the automorphism invariants of $A_{\vec{r}}^{(1)}, \vec{r}=\left(r_{1}, \ldots, r_{s}\right)$. The main ideas however are present in the much simpler special case $s=1$. In order to make the general argument clearer, in this section we will limit the discussion to $A_{r}^{(1)}$.

The proof is surprisingly simple. Calculus will show that the q-dimensions eq.(3b) force the automorphism invariant $\sigma$ to map the weight $\omega_{1}=(k-1) \Lambda_{0}+\Lambda_{1}$ to $C_{r}^{a} J_{r}^{b} \omega_{1}$, for some $a, b$. Using eq.(5b) we will see that $\sigma \omega_{1}=C_{r}^{a} \sigma_{m} \omega_{1}$ for some known automorphism invariant $\sigma_{m}$. Thus it suffices to consider the case where $\sigma \omega_{1}=\omega_{1}$. Using Verlinde's formula, we can then show that $\sigma$ must fix all $\omega_{i}=(k-1) \Lambda_{0}+\Lambda_{i}$. But any Weyl character $c h_{\bar{\lambda}}$ is a polynomial in the $c h_{\overline{\omega_{i}}}$ - from eq.(3a) this tells us $\sigma$ must fix all weights.

Because of eqs.(4c),(4d) we can expect to build automorphism invariants from $J_{r}$. In particular [25], define

$$
\tilde{k}=\left\{\begin{array}{cc}
\bar{k} & \text { if } r \equiv \bar{k} \equiv 0 \quad(\bmod 2) \\
k & \text { otherwise }
\end{array}\right.
$$


Choose any positive integer $m$ dividing $\bar{r}$ such that $m \tilde{k}$ is even and $\operatorname{gcd}\{\bar{r} / m, m \tilde{k} / 2\}=1$. Then we can find an integer $v$ such that $v m \tilde{k} / 2 \equiv 1(\bmod \bar{r} / m)$. To each such divisor $m$ of $\bar{r}$, we can define an automorphism invariant $\sigma_{m}$ given by

$$
\sigma_{m} \lambda \stackrel{\text { def }}{=} J_{r}^{-v m t(\lambda)} \lambda \text {. }
$$

For example, $\sigma_{\bar{r}}$ corresponds to the identity permutation. That each $\sigma_{m}$ is a bijection of $P_{+}^{r, k}$, follows from $\sigma_{m} \circ \sigma_{m}=i d$.. That they satisfy eqs.(5) can be verified explicitly, using eqs.(4).

Theorem 1 The only automorphism invariants $\sigma$ of $A_{r}^{(1)}$ at level $k$ are $C_{r}^{a} \sigma_{m}$, for $a=0,1$ and $\sigma_{m}$ defined in eq.(6b). All of these are distinct, except for $r=1$ (when $C_{r}=i d$.) or $k \leq 2$.

Remark 1 From Theorem 1 we find that there are precisely $2^{c+p+t}$ automorphism invariants for $A_{r}^{(1)}$ at level $k$, where:

$c=1$, unless $r=1$ or $k \leq 2$ when $c=0$ (an exception: $c=-1$ when both $r=1$ and $k=2)$;

$p$ is the number of distinct odd primes which divide $\bar{r}$ but not $k$;

$t=0$ if either $r$ is even, or $r$ is odd and $k \equiv 0(\bmod 4)$, or $k$ is odd and $r \equiv 1(\bmod 4)$ - otherwise $t=1$.

Remark 2 From the theorem we also see that all automorphism invariants are of order 2 (i.e. $\sigma^{2}=i d$.), and commute: in fact

$$
C_{r}^{a} \sigma_{m} \circ C_{r}^{b} \sigma_{m^{\prime}}=C_{r}^{a+b} \sigma_{m^{\prime \prime}}, \text { where } m^{\prime \prime}=\bar{r} g c d\left\{m, m^{\prime}\right\}^{2} / m m^{\prime} .
$$

Both of these facts are surprising, and not true for general $g$ (as we will see in Section 3).

Three special cases of the theorem were known previously: $r=1[5], k=1$ [16], and $r=2[10,24]$. The remainder of this section is devoted to the proof of Theorem 1 .

For each $i=1, \ldots, r$ define $\omega_{i}=(k-1) \Lambda_{0}+\Lambda_{i}$. For any $\lambda$ define the Aut $\Pi^{\vee}$-orbit $[\lambda]=\left\{C_{r}^{a} J_{r}^{b} \lambda \mid a, b \in \mathbf{Z}\right\}$. Let $o(\lambda)$ equal the number of indices $0 \leq i \leq r$ such that $\lambda_{i}>0$. For example, $\lambda \in\left[k \Lambda_{0}\right]$ iff $o(\lambda)=1$. Also, $o\left(\omega_{i}\right)=2$ (provided $k>1$ ).

Let us begin by proving the second statement in the Theorem. That the $\sigma_{m}$ 's are all distinct is easy to see by looking at $\sigma_{m} \omega_{1}$. Next, for $r>1$ and $k>2, C_{r} \omega_{1} \notin\left\{J_{r}^{i} \omega_{1} \mid i \in \mathbf{Z}\right\}$, so $C_{r} \sigma_{m} \omega_{1} \neq \sigma_{m^{\prime}} \omega_{1}$ for any $m, m^{\prime}$. Incidently, when $k \leq 2, C_{r}=\sigma_{m}$ where $m=1$ or 2 .

Recall $Q^{r, k}(\lambda)$ defined in eq.(3b). It will be constant within each orbit $[\lambda]$. Eq.(3b) was analysed in [8], by extending the domain of $Q$ to real (as opposed to integer) vectors $\lambda$. We will make use of this idea below.

Proposition 1 For $k>1$ and any $\lambda \notin\left[k \Lambda_{0}\right] \cup\left[\omega_{1}\right], Q^{r, k}(\lambda)>Q^{r, k}\left(\omega_{1}\right)>Q^{r, k}\left(k \Lambda_{0}\right)$. Proof. Consider any $\lambda \in P_{+}^{r, k}, \lambda \notin\left[k \Lambda_{0}\right]$. Suppose first that $o(\lambda) \geq 3$, and let $\lambda_{i}, \lambda_{j}>0$ for $i \neq j>0$. Consider $\mu(t)=\lambda+t \Lambda_{j}-t \Lambda_{i}$. Then $\left(\mu\left(\lambda_{i}\right)\right)_{i}=\left(\mu\left(-\lambda_{j}\right)\right)_{j}=0$. An easy calculation, similar to one in [8], tells us

$$
\left.\frac{\mathrm{d}}{\mathrm{d} t} Q(\mu(t))\right|_{t=t_{0}}=\left.0 \Rightarrow \frac{\mathrm{d}^{2}}{\mathrm{~d} t^{2}} Q(\mu(t))\right|_{t=t_{0}}=-Q\left(\mu\left(t_{0}\right)\right) \frac{\pi^{2}}{\bar{k}^{2}} \sum_{\bar{\alpha}>0} \frac{(\bar{a} \mid \bar{\alpha})^{2}}{\sin ^{2}\left[\pi\left(\overline{\mu\left(t_{0}\right)+\rho} \mid \bar{\alpha}\right) / \bar{k}\right]}<0
$$


where $\bar{a}=\overline{\Lambda_{j}}-\overline{\Lambda_{i}}$. Thus $Q(\mu(t))$ will take its minimum on the endpoints, i.e. either $Q\left(\mu\left(\lambda_{i}\right)\right)<Q(\lambda)$ or $Q\left(\mu\left(-\lambda_{j}\right)\right)<Q(\lambda)$. In either case, we have found a $\mu \in P_{+}^{r, k}$ with $Q(\mu)<Q(\lambda)$, which has $o(\mu)=o(\lambda)-1$.

Continuing inductively, we see it suffices to consider the weights with $o(\lambda)=2$. The same argument allows us to put one of those two Dynkin labels equal to 1 . In other words, starting with any weight $\lambda \notin\left[k \Lambda_{0}\right]$, we can find a $\omega_{\ell}$ such that $Q(\lambda) \geq Q\left(\omega_{\ell}\right)$, with equality iff $\lambda \in\left[\omega_{\ell}\right]$. All that remains is to compare $Q\left(\omega_{\ell}\right)$ with $Q\left(\omega_{1}\right)$.

We find from eq. $(3 \mathrm{~b})$ that for all $1<m \leq r$,

$$
\frac{Q\left(\omega_{m}\right)}{Q\left(\omega_{m-1}\right)}=\prod_{i=1}^{m-1} \frac{\sin \left[\pi \frac{m-i}{\bar{k}}\right]}{\sin \left[\pi \frac{m+1-i}{\bar{k}}\right]} \prod_{j=m}^{r} \frac{\sin \left[\pi \frac{j+2-m}{\bar{k}}\right]}{\sin \left[\pi \frac{j+1-m}{\bar{k}}\right]}=\frac{\sin \left[\pi \frac{r+2-m}{\bar{k}}\right]}{\sin \left[\pi \frac{m}{\bar{k}}\right]}
$$

Hence $Q\left(\omega_{1}\right)<Q\left(\omega_{\ell}\right)$ unless $\ell=1$ or $r$. But $\omega_{r}=C_{r} \omega_{1} \in\left[\omega_{1}\right]$. Finally, compute $Q\left(\omega_{1}\right)=\sin [\pi \bar{r} / \bar{k}] / \sin [\pi / \bar{k}] \geq 1=Q\left(k \Lambda_{0}\right)$, with equality only at $k=1$. QED

The restriction in Prop. 1 to $k>1$ is not important, because $P_{+}^{r, 1}=\left[k \Lambda_{0}\right]$. Prop. 1 together with eq.(5a) tells us, for any $r \geq 1, k \geq 1$, that $\sigma \omega_{1}=C_{r}^{a} J_{r}^{b} \omega_{1}$ for some $a, b$. By replacing $\sigma$ with $C_{r}^{a} \sigma$, we may assume $a=0$. Evaluating the expression $T_{\omega_{1}, \omega_{1}}^{r, k}=T_{J_{r}^{b} \omega_{1}, J_{r}^{b} \omega_{1}}^{r, k}$ using eq.(4c), we get

$$
2 b \equiv k b(\bar{r}-b) \quad(\bmod 2 \bar{r}) .
$$

Proposition 2 Let $b$ be any integer satisfying eq.( ${ }^{7}$ ( $\left.a\right)$. Then $\sigma_{m}\left(J_{r}^{b} \omega_{1}\right)=\omega_{1}$, for one of the $\sigma_{m}$ of eq. $(6 b)$.

Proof. Given any solution $b$ to eq.(7a), define $m=\operatorname{gcd}\{b, \bar{r}\}$ and $v^{\prime}=b / m$ - we can suppose (adding $\bar{r}$ to $b$ if necessary) that $v^{\prime}$ will be coprime to $2 \bar{r} / m$. Suppose we can show

$$
\tilde{k} b / 2 \equiv-1 \quad(\bmod \bar{r} / m) .
$$

Then $\tilde{k} m / 2$ will be an integer coprime to $\bar{r} / m$ (so that $\sigma_{m}$ exists), and $b \equiv-v m(\bmod \bar{r})$ for $v$ as in eq. $(6 \mathrm{~b})$ (so that $\left.\sigma_{m}\left(J_{r}^{b} \omega_{1}\right)=\omega_{1}\right)$.

Now, dividing eq. (7a) by $b$ gives

$$
k b \equiv-2+k \bar{r} \quad(\bmod 2 \bar{r} / m) .
$$

For $\bar{r}$ odd, $b$ is odd and eq.(7b) is immediate from eq.(7c). For $\bar{r}$ even, eq.(7c) requires either $k$ or $m$ to be even, so again eq.(7b) follows from eq.(7c). QED

By Prop. 2 we see that, replacing $\sigma$ with $\sigma_{m} \circ \sigma$, it suffices to consider those $\sigma$ fixing $\omega_{1}$. Theorem 1 is proved if we can show such a $\sigma$ must equal the identity.

A convenient way to exploit eq.(3a) is to use the fusion rules, which we may take to be defined by Verlinde's formula [26]:

$$
N_{\lambda, \mu}^{\nu} \stackrel{\text { def }}{=} \sum_{\beta \in P_{+}^{r, k}} S_{\lambda, \beta} \frac{S_{\mu, \beta}}{S_{k \Lambda_{0}, \beta}} S_{\nu, \beta}^{*}
$$


These $N_{\lambda, \mu}^{\nu}$ have a well-known geometric interpretation, but it is irrelevant for our purposes. Kac ([18]; see also p.288 of [17]) and Walton [28] used eq.(3a) to reduce eq.(8a) to an expression involving finite-dimensional tensor product decompositions. In particular, for any $\omega$ in the affine Weyl group $W$ of $A_{r}^{(1)}$, let $\epsilon(\omega)$ denote the sign of $\omega$, and $\omega \cdot \lambda=$ $\omega(\lambda+\rho)-\rho$. Let mult $_{\bar{\lambda} \otimes \bar{\mu}}(\bar{\nu})$ denote the multiplicity (i.e. the Littlewood-Richardson coefficient) of the $A_{r}$-module $\bar{L}(\bar{\nu})$ in $\bar{L}(\bar{\lambda}) \otimes \bar{L}(\bar{\mu})$. Then we find

$$
N_{\lambda, \mu}^{\nu}=\sum_{\omega \in W} \epsilon(\omega) \operatorname{mult}_{\bar{\lambda} \otimes \bar{\mu}}(\overline{\omega \cdot \nu})
$$

We will use in Section 3 the following facts, obvious from eqs.(8a),(4d),(4e):

$$
\begin{gathered}
N_{\lambda, k \Lambda_{0}}^{\nu}=\delta_{\lambda, \nu}, \quad \forall \lambda, \nu \in P_{+}^{r, k} \\
N_{C_{r}^{c} J_{r}^{a} \lambda, C_{r}^{c} J_{r}^{b} \mu}^{C_{r}^{a+b}}=N_{\lambda, \mu}^{\nu}, \quad \forall \lambda, \mu, \nu \in P_{+}^{r, k}, \forall a, b, c \in \mathbf{Z} .
\end{gathered}
$$

From eqs.(5a),(8a), $\sigma$ must be a symmetry of the fusion rules:

$$
N_{\lambda, \mu}^{\nu}=N_{\sigma \lambda, \sigma \mu}^{\sigma \nu}
$$

It is thus natural to look at the fusions involving $\omega_{1}$ : from eq.(8b) we find

$$
N_{\lambda, \omega_{1}}^{\nu}=\left\{\begin{array}{cc}
1 & \text { if } \nu \in \lambda+\left\{\Lambda_{1}-\Lambda_{0}, \Lambda_{2}-\Lambda_{1}, \ldots, \Lambda_{0}-\Lambda_{r}\right\} \\
0 & \text { otherwise }
\end{array} .\right.
$$

Now suppose $\sigma \omega_{j}=\omega_{j}$ for all $1 \leq j \leq i<r$. From eq.(9b), $N_{\omega_{i}, \omega_{1}}^{\nu}=1$ iff either $\nu=\omega_{i+1}$ or (for $\left.k>1\right) \nu=\omega_{i}^{\prime} \stackrel{\text { def }}{=}(k-2) \Lambda_{0}+\Lambda_{1}+\Lambda_{i}$. This means, from eq.(9a), that $\sigma \omega_{i+1}$ must equal either $\omega_{i+1}$ or $\omega_{i}^{\prime}$. But

$$
\left(\omega_{i}^{\prime}+\rho \mid \omega_{i}^{\prime}+\rho\right)-\left(\omega_{i+1}+\rho \mid \omega_{i+1}+\rho\right)=2 i+2 \not \equiv 0 \quad(\bmod 2 \bar{k}) .
$$

Therefore by eq.(5b), $\sigma \omega_{i+1}=\omega_{i+1}$.

Thus by induction $\sigma$ must fix each $\omega_{1}, \ldots, \omega_{r}$. From this we can now complete the proof of the theorem, with the following observation:

Proposition 3 Suppose we have weights $\lambda, \lambda^{\prime} \in P_{+}^{r, k}$ such that

$$
\frac{S_{\omega_{i}, \lambda}}{S_{k \Lambda_{0}, \lambda}}=\frac{S_{\omega_{i}, \lambda^{\prime}}}{S_{k \Lambda_{0}, \lambda^{\prime}}}
$$

for all $i$. Then $\lambda=\lambda^{\prime}$.

Proof. We know (see Ch.VI, $§ 3.4$, Th.1 of [3]) that the Weyl character $\operatorname{ch}_{\bar{\beta}}$ of the $\bar{g}$ module $\bar{L}(\bar{\beta})$ of any finite dimensional Lie algebra $\bar{g}$ can be written as a polynomial $p_{\bar{\beta}}$ of $c h_{\overline{\omega_{1}}}, \ldots, c h \overline{\omega_{r}}$. From eq.(3a) we thus get

$$
\frac{S_{\beta, \gamma}}{S_{k \Lambda_{0}, \gamma}}=p_{\bar{\beta}}\left(\frac{S_{\omega_{1}, \gamma}}{S_{k \Lambda_{0}, \gamma}}, \ldots, \frac{S_{\omega_{r}, \gamma}}{S_{k \Lambda_{0}, \gamma}}\right), \quad \forall \beta, \gamma \in P_{+}^{r, k}
$$


Equations (10a),(10b) together tell us that in fact

$$
\frac{S_{\beta, \lambda}}{S_{k \Lambda_{0}, \lambda}}=\frac{S_{\beta, \lambda^{\prime}}}{S_{k \Lambda_{0}, \lambda^{\prime}}} \quad \forall \beta \in P_{+}^{r, k}
$$

Multiplying it by $S_{\beta, \lambda}^{*}$ and summing over all $\beta$ forces $\lambda=\lambda^{\prime}$ by unitarity of $S$.

QED

Choose any $\lambda \in P_{+}^{r, k}$ and put $\lambda^{\prime}=\sigma \lambda$. Then from eq.(5a) and using $\sigma\left(k \Lambda_{0}\right)=k \Lambda_{0}$ and $\sigma \omega_{i}=\omega_{i}$ we find that eq.(10a) is satisfied. Then Prop. 3 tells us $\lambda=\lambda^{\prime}$. In other words, $\sigma$ must be the identity, and Theorem 1 is proved.

\section{The automorphism invariants of $\left(A_{r_{1}} \oplus \cdots \oplus A_{r_{s}}\right)^{(1)}$}

The affinization of reductive Lie algebras is discussed e.g. in $\S \S 12.9-12.10$ of [17]. Of course most quantities for semi-simple Lie algebras can be built up in a straightforward way from those for the simple ones. But this is not true for automorphism invariants, as we shall see. Knowing the list of automorphism invariants for the affinization of simple algebras $\bar{g}_{i}$ helps very little in their classification for the affinization of $\bar{g}_{1} \oplus \cdots \oplus \bar{g}_{s}$. Nevertheless, with some additional complications the techniques developed in the last section can be applied to the classification of automorphism invariants for $A_{\vec{r}}^{(1)}$. Only one case with $s>1$ was known previously: all $r_{i}=1$ [11].

Theorem 2 gives the classification and is the main result of this section. We would have liked to find an explicit set of generators for the group of automorphism invariants, but this seems to be more work than it is worth, for general $r_{i}$. Instead we will limit ourselves here to two special cases, which together form Theorem 3.

The level here is an $s$-tuple $k=\left(k_{1}, \ldots, k_{s}\right)$, each $k_{i}$ a positive integer. Write $r=\vec{r}=$ $\left(r_{1}, \ldots, r_{s}\right)$. As before call $\bar{r}_{i}=r_{i}+1, \bar{k}_{i}=k_{i}+\bar{r}_{i}$. The set of highest weights is

$$
P_{+}^{r, k} \stackrel{\text { def }}{=} P_{+}\left(A_{\vec{r}}^{(1)}, k\right) \bmod \mathbf{C} \delta=\left\{\lambda=\sum_{i=1}^{s} \lambda_{(i)} \mid \lambda_{(i)}=\sum_{j=0}^{r_{i}} \lambda_{(i) j} \Lambda_{j}^{i} \in P_{+}^{r_{i}, k_{i}}\right\},
$$

using obvious notation. The modular matrix $S$ will be

$$
S_{\lambda, \mu}^{r, k}=\prod_{i=1}^{s} S_{\lambda_{(i)}, \mu_{(i)}}^{r_{i}, k_{i}}
$$

similarly for $T^{r, k}$. We will usually drop the superscripts ' $r, k$ '. For $a=\left(a_{1}, \ldots, a_{s}\right)$, write

$$
\begin{gathered}
J_{r}^{a} \lambda \stackrel{\text { def }}{=} \sum_{i=1}^{s} J_{r_{i}}^{a_{i}} \lambda_{(i)}, \\
C_{r}^{a} \lambda \stackrel{\text { def }}{=} \sum_{i=1}^{s} C_{r_{i}}^{a_{i}} \lambda_{(i)},
\end{gathered}
$$


and $C_{r}=C_{r_{1}} \cdots C_{r_{s}}$. Let $k \Lambda_{0}=\sum_{j} k_{j} \Lambda_{0}^{j}$, and for each $1 \leq i \leq s, 1 \leq \ell \leq r_{i}$, define

$$
\omega_{\ell}^{i} \stackrel{\text { def }}{=} \sum_{j \neq i} k_{j} \Lambda_{0}^{j}+\left(k_{i}-1\right) \Lambda_{0}^{i}+\Lambda_{\ell}^{i} \in P_{+}^{r, k}
$$

Finally, write $[\lambda]$ for the orbit $\left\{C_{r}^{a} J_{r}^{b} \lambda \mid a_{i}, b_{i} \in \mathbf{Z}\right\}$, and $Q(\lambda)=S_{\lambda, k \Lambda_{0}} / S_{k \Lambda_{0}, k \Lambda_{0}}$. Prop. 1 implies

$$
Q(\lambda)=1 \text { iff } \lambda \in\left[k \Lambda_{0}\right]
$$

Examples of automorphism invariants are the $C_{r}^{a}$ of eq.(11a). Another example is induced by any permutation $\pi$ of the indices $\{1, \ldots, s\}$ with the property that

$$
k_{i}=k_{\pi i}, \quad r_{i}=r_{\pi i}, \quad \forall i
$$

the corresponding automorphism invariant is the map $\sigma_{\pi}$ defined by

$$
\sigma_{\pi} \lambda=\sum_{i=1}^{s} \lambda_{(\pi i)}
$$

Find any integers $a_{i j}$, for all $1 \leq i, j \leq s$, satisfying:

$$
\begin{aligned}
& a_{i j} \bar{r}_{i} / \bar{r}_{j} \in \mathbf{Z} \quad \forall i, j ; \\
& \frac{2 a_{i i}}{\bar{r}_{i}}+\sum_{j=1}^{s} k_{j} \frac{a_{i j}^{2}}{\bar{r}_{j}} \equiv \sum_{j=1}^{s} k_{j} a_{i j} \quad(\bmod 2), \quad \forall i \\
& \frac{a_{i j}}{\bar{r}_{j}}+\frac{a_{j i}}{\bar{r}_{i}}+\sum_{\ell=1}^{s} k_{\ell} \frac{a_{i \ell} a_{j \ell}}{\bar{r}_{\ell}} \equiv 0 \quad(\bmod 1), \quad \forall i, j .
\end{aligned}
$$

To any such matrix $a$, define a function $\sigma_{a}$ on $P_{+}^{r, k}$ by

$$
\sigma_{a} \lambda=J_{r}^{t(\lambda) a} \lambda \stackrel{\text { def }}{=} \sum_{j=1}^{s} \prod_{i=1}^{s} J_{r_{j}}^{a_{i j} t\left(\lambda_{(i)}\right)} \lambda_{(j)}
$$

Because of eq.(13a), $\sigma_{a}$ will be a permutation, with inverse $\sigma_{b}$ where $b_{i j}=\bar{r}_{j} a_{j i} / \bar{r}_{i}$. Because of eqs.(13b),(13c), $\sigma_{a}$ will satisfy eqs.(5) and be an automorphism invariant. Note that

$$
\pi_{b} \circ \pi_{a}=\pi_{c}, \quad \text { where } c_{i j} \equiv a_{i j}+b_{i j}+\sum_{\ell=1}^{s} k_{\ell} a_{i \ell} b_{\ell j} \quad\left(\bmod \bar{r}_{j}\right) \text {. }
$$

Theorem $2 \quad \sigma$ is an automorphism invariant of $A_{\vec{r}}^{(1)}$ at level $k=\left(k_{1}, \ldots, k_{s}\right)$ iff $\sigma=$ $\sigma_{\pi} \circ C_{r}^{c} \circ \sigma_{a}$, where $\sigma_{\pi}$ is defined in eq.(12b), $C_{r}^{c}$ in eq.(11a), and $\sigma_{a}$ in eq.(13d). 
Remark. Note that in general we have here neither $\sigma^{2}=i d$. nor $\sigma \circ \sigma^{\prime}=\sigma^{\prime} \circ \sigma$. Also, we have here many more solutions than could be built by tensoring together $s=1$ automorphism invariants. Provided we demand $c_{i}=c_{i}^{\prime}=0$ when $r_{i}=1$ or $k_{i} \leq 2$, and $\pi i=\pi^{\prime} i=i$ when $k_{i}=1$, then

$$
\sigma_{\pi} \circ C_{r}^{c} \circ \sigma_{a}=\sigma_{\pi^{\prime}} \circ C_{r}^{c^{\prime}} \circ \sigma_{a^{\prime}} \Leftrightarrow \pi i=\pi^{\prime} i, c_{i} \equiv c_{i}^{\prime}(\bmod 2), a_{i j} \equiv a_{i j}^{\prime}\left(\bmod \bar{r}_{j}\right) \forall i, j .
$$

Proof. Write $Q_{i}=Q\left(\omega_{1}^{i}\right)$. For convenience reorder the indices so that $Q_{1} \leq Q_{2} \leq \cdots \leq$ $Q_{s}$. We will begin by finding an index $\pi i$ for each $i$, so that $\sigma \omega_{1}^{i} \in\left[\omega_{1}^{\pi i}\right]$. As in eq.(8a) put

$$
N_{\lambda, \mu}^{\nu} \stackrel{\text { def }}{=} \sum_{\beta \in P_{+}^{r, k}} S_{\lambda, \beta}^{r, k} \frac{S_{\mu, \beta}^{r, k}}{S_{k \Lambda_{0}, \beta}^{r, k}} S_{\nu, \beta}^{r, k *}=\prod_{i=1}^{s} N_{\lambda_{(i)}, \mu_{(i)}}^{\nu_{(i)}}
$$

Let $o_{i}(\lambda)$ equal the number of $0 \leq j \leq r_{i}$ such that $\lambda_{(i) j}>0$. Then from eqs.(9b),(8c),

$$
\sum_{\nu \in P_{+}^{r, k}} N_{\lambda, \omega_{1}^{i}}^{\nu}=o_{i}(\lambda), \quad \forall i \in\{1, \ldots, s\}, \lambda \in P_{+}^{r, k}
$$

We will construct $\pi$ by induction on $i$. Suppose for all $j<i$, we have a $\pi j$ such that $\sigma \omega_{1}^{j} \in\left[\omega_{1}^{\pi j}\right]$. By eqs.(8d),(14a),(14b),(5a) we know for these $j<i$ that

$$
o_{j}(\lambda)=o_{\pi j}(\sigma \lambda), \quad \forall \lambda \in P_{+}^{r, k}
$$

If $Q_{i}=1$ (i.e. $k_{i}=1$ ), then both $\omega_{1}^{i}, \sigma \omega_{1}^{i} \in\left[k \Lambda_{0}\right]$ by eqs.(5a),(11b), so put $\pi i=i$ there $\left(k_{i}=1\right.$ is a special - albeit trivial - case here because for those $i, \pi i$ is not fixed by the constraint $\left.\sigma \omega_{1}^{i} \in\left[\omega_{1}^{\pi i}\right]\right)$. When $Q_{i}>1,\left(\sigma \omega_{1}^{i}\right)_{(j)} \in\left[k \Lambda_{0}\right]_{(j)}$ for all $j$ with $Q_{j}<Q_{i}$, by eq.(14c). So by Prop. 1 , we must have $\sigma \omega_{1}^{i} \in\left[\omega_{1}^{\ell}\right]$ for some $\ell$ with $Q_{i}=Q_{\ell}$. Put $\pi i=\ell$.

This inductively defines $\pi i$ for all $i$, in such a way that $\sigma \omega_{1}^{i} \in\left[\omega_{1}^{\pi i}\right]$. Next, let us show $\pi$ is a bijection. It suffices to show that $\pi$ is onto. Choose any $1 \leq \ell \leq s$ - we may suppose $k_{\ell}>1$. Because $\sigma^{-1}$ is also an automorphism invariant, there exists an $i$ such that $\sigma\left(C_{r}\right)^{a} J_{r}^{b} \omega_{1}^{i}=\omega_{1}^{\ell}$ for some $a \in\{0,1\}, b=\left(b_{1}, \ldots, b_{s}\right)$.

Now $\sigma$ commutes with $S$, so it also does with $C^{r, k}=\left(S^{r, k}\right)^{2}$. Also, by eqs.(5a),(11b) $\sigma J_{r}^{b}\left(k \Lambda_{0}\right)=J_{r}^{c}\left(k \Lambda_{0}\right)$ for some $c$. But because of eqs.(4d),(3c),(5a) we know

$$
S_{J_{r}^{b} \lambda, \mu}=S_{\lambda, \mu} \frac{S_{J_{r}^{b}\left(k \Lambda_{0}\right), \mu}}{S_{k \Lambda_{0}, \mu}}=S_{\sigma \lambda, \sigma \mu} \frac{S_{J_{r}^{c}\left(k \Lambda_{0}\right), \sigma \mu}}{S_{k \Lambda_{0}, \sigma \mu}}=S_{J_{r}^{c} \sigma \lambda, \sigma \mu}, \quad \forall \lambda, \mu \in P_{+}^{r, k} .
$$

Therefore by unitarity of $S, \sigma J_{r}^{b}=J_{r}^{c} \sigma$. Hence $\sigma \omega_{1}^{i}=J_{r}^{-c}\left(C_{r}\right)^{a} \omega_{1}^{\ell} \in\left[\omega_{1}^{\ell}\right]$, so $\pi i=\ell$, and $\pi$ is a bijection.

Finally we want to show $\pi$ satisfies eq.(12a). Write $\sigma \omega_{1}^{i}=\left(C_{r}\right)^{c_{i}} J_{r}^{d} \omega_{1}^{\pi i}$, for some $c_{i} \in\{0,1\}, d=\left(d_{1}, \ldots, d_{s}\right)$. The expression

$$
1=N_{\omega_{1}^{i}, \omega_{1}^{i}}^{\lambda}=N_{\left(C_{r}\right)^{c_{i}} J_{r}^{d} \omega_{1}^{\pi i},\left(C_{r}\right)^{c_{i}} J_{r}^{d} \omega_{1}^{\pi i}}^{\sigma \lambda}
$$


requires from eqs.(14a),(8c),(8d),(9b) that $\lambda=\omega_{2}^{i}$ or $\lambda=\omega_{1}^{i \prime} \stackrel{\text { def }}{=} \sum_{j \neq i} k_{j} \Lambda_{0}^{j}+\left(k_{i}-2\right) \Lambda_{0}^{i}+$ $2 \Lambda_{1}^{i}$, and that $\sigma \lambda=\left(C_{r}\right)^{c_{i}} J_{r}^{2 d} \omega_{2}^{\pi i}$ or $\sigma \lambda=\left(C_{r}\right)^{c_{i}} J_{r}^{2 d} \omega_{1}^{\pi i \prime}$. Thus either:

(i) $\sigma \omega_{2}^{i}=\left(C_{r}\right)^{c_{i}} J_{r}^{2 d} \omega_{2}^{\pi i}$ and $\sigma \omega_{1}^{i \prime}=\left(C_{r}\right)^{c_{i}} J_{r}^{2 d} \omega_{1}^{\pi i \prime}$; or

(ii) $\sigma \omega_{2}^{i}=\left(C_{r}\right)^{c_{i}} J_{r}^{2 d} \omega_{1}^{\pi i \prime}$ and $\sigma \omega_{1}^{i \prime}=\left(C_{r}\right)^{c_{i}} J_{r}^{2 d} \omega_{2}^{\pi i}$.

But from eqs. (5b), (4c) and using $\sigma \omega_{1}^{i}=\left(C_{r}\right)^{c_{i}} J_{r}^{d} \omega_{1}^{\pi i}$, case (i) is seen to require that

$$
\frac{\bar{r}_{i} \pm 1}{\bar{k}_{i}} \equiv \frac{\bar{r}_{\pi i} \pm 1}{\bar{k}_{\pi i}} \quad(\bmod 1)
$$

while case (ii) requires

$$
\frac{\bar{r}_{i} \pm 1}{\bar{k}_{i}} \equiv \frac{\bar{r}_{\pi i} \mp 1}{\bar{k}_{\pi i}} \quad(\bmod 1) .
$$

Since $2 \leq \bar{r}_{j} \leq \bar{k}_{j}-1$ for all $j$, eq.(15a) forces eq.(12a) while eq.(15b) has no solution.

Thus $\pi$ satisfies eq.(12a). Replacing $\sigma$ by $\sigma_{\pi^{-1}} \circ \sigma$, we may assume $\sigma \omega_{1}^{i} \in\left[\omega_{1}^{i}\right]$ for all $i$. Likewise, by replacing $\sigma$ with $C_{r}^{c} \sigma$ for some $c=\left(c_{1}, \ldots, c_{s}\right)$ (we can require $c_{i}=0$ when $r_{i}=1$ or $k_{i} \leq 2$ ), we may suppose, for each $i$, that

$$
\sigma \omega_{1}^{i}=J_{r}^{a_{i}} \omega_{1}^{i}, \quad \text { where } a_{i}=\left(a_{i 1}, \ldots, a_{i s}\right)
$$

Eqs.(5a),(4d) and (5b),(4c) give us eqs.(13c),(13b) respectively. Using a calculation given earlier this proof, $\sigma^{-1}$ will be an automorphism invariant satisfying eq.(16a) for some matrix $b$ in place of $a$. Looking at $S_{\omega_{1}^{i}, \sigma^{-1} \omega_{1}^{j}}=S_{\sigma \omega_{1}^{i}, \omega_{1}^{j}}$ we find from eq.(4d)

$$
b_{j i} / \bar{r}_{i} \equiv a_{i j} / \bar{r}_{j} \quad(\bmod 1),
$$

thus eq.(13a) will also be satisfied. This means $\sigma_{a}$ defines an automorphism invariant; replacing $\sigma$ with $\sigma_{a}^{-1} \circ \sigma$, we may then assume all $a_{i j}=0$. It suffices now to prove any such $\sigma$ must be the identity.

By exactly the same induction argument used in Section 2, together with eqs.(8c),(14a), (9b) the expression

$$
1=N_{\omega_{i}^{j}, \omega_{1}^{j}}^{\omega_{i+1}^{j}}=N_{\omega_{i}^{j}, \omega_{1}^{j}}^{\sigma \omega_{i+1}^{j}}
$$

gives only two possibilities for $\sigma \omega_{i+1}^{j}$. As before, only $\sigma \omega_{i+1}^{j}=\omega_{i+1}^{j}$ satisfies eq.(5b). This argument shows that $\sigma$ must fix all $\omega_{m}^{\ell}$. The proof of Prop. 3 now carries over without change, and we find that indeed $\sigma \lambda=\lambda$ for all $\lambda \in P_{+}^{r, k}$. QED

As is done in Section 2 for the special case $s=1$, it should be possible to find a complete set of generators for the group of automorphism invariants of $A_{\vec{r}}^{(1)}$ at fixed level $k$, as well as compute its order. But both of these will be messy, depending on $r$ and $k$ in a more complicated way than is the case for $s=1$. We will limit ourselves here to two simple observations (see Theorem 3 below).

But first, define four new types of automorphism invariants $\sigma\left[J_{r}^{m}\right], \sigma[p ; \ell, m], \sigma[\ell, m, n]$ and $\sigma[\ell, m, n, o]$ as follows. 
(i) Choose any $m=\left(m_{1}, \ldots, m_{s}\right)$. Define $\tilde{k}_{i}=\bar{k}_{i}$ or $k_{i}$ as in eq.(6a). Put $u=$ $\sum_{i} \tilde{k}_{i} m_{i}\left(\bar{r}_{i}-m_{i}\right) / \bar{r}_{i}, \bar{m}_{i}=\bar{r}_{i} / g c d\left\{m_{i}, \bar{r}_{i}\right\}$, and $N=l c m_{i}\left\{\bar{m}_{i}\right\}$. Suppose $N u$ is even, and $N u / 2$ is coprime to $N$. Then there exists an integer $v$ such that $v N u / 2 \equiv 1(\bmod$ $N$ ). Define $\sigma\left[J_{r}^{m}\right]=\sigma_{a}$, where $a_{i j}=v N m_{j} m_{i} / \bar{r}_{i}$ (this is the immediate generalization of eq.(6b)).

(ii) Choose any prime $p$, and any indices $1 \leq \ell<m \leq s$ such that $p$ divides $\bar{r}_{\ell}, k_{\ell}, \bar{r}_{m}$ and $k_{m}$. If $p=2$ we need the additional constraint that 8 divides both $\bar{r}_{\ell} k_{\ell}$ and $\bar{r}_{m} k_{m}$. Define $\sigma[p ; \ell, m]$ to be $\sigma_{a}$, where $a_{i j}=0$ for all $i, j$ except for $a_{\ell m}=\bar{r}_{m} / p$, $a_{m \ell}=-\bar{r}_{\ell} / p$.

(iii) Find distinct indices $\ell, m, n$ with $k_{\ell}, k_{m}$ both odd, $k_{n} \equiv 0(\bmod 4)$, and $\bar{r}_{\ell} \equiv \bar{r}_{m} \equiv$ $\bar{r}_{n} \equiv 2(\bmod 4)$, such that $k_{\ell} \bar{r}_{\ell}+k_{m} \bar{r}_{m} \equiv 0(\bmod 8)$. Define $\sigma[\ell, m, n]$ to be $\sigma_{a}$, where $a_{i j}=0$ except for $a_{\ell n}=a_{m n}=\bar{r}_{n} / 2, a_{n \ell}=\bar{r}_{\ell} / 2$, and $a_{n m}=\bar{r}_{m} / 2$.

(iv) Find distinct indices $\ell, m, n, o$, with $k_{\ell}, k_{m}, k_{n}, k_{o}$ all odd, and $\bar{r}_{\ell} \equiv \bar{r}_{m} \equiv \bar{r}_{n} \equiv \bar{r}_{o} \equiv 2$ $(\bmod 4)$, such that $k_{m} \bar{r}_{m}+k_{n} \bar{r}_{n} \equiv k_{\ell} \bar{r}_{\ell}+k_{o} \bar{r}_{o} \equiv 0(\bmod 8)$. Define $\sigma[\ell, m, n, o]$ to be $\sigma_{a}$, where $a_{i j}=0$ except for $a_{\ell m}=a_{o m}=\bar{r}_{m} / 2, a_{\ell n}=a_{o n}=\bar{r}_{n} / 2, a_{m \ell}=a_{n \ell}=\bar{r}_{\ell} / 2$ and $a_{m o}=a_{n o}=\bar{r}_{o} / 2$.

Theorem 3 (a) If $\operatorname{gcd}\left\{2 k_{i}, \bar{r}_{i}\right\}=1$ for each $i$, then the $\sigma\left[J_{r}^{m}\right]$ of (i) above, together with $C_{r}^{c}$ and $\sigma_{\pi}$, generate the group of all automorphism invariants of $A_{\vec{r}}^{(1)}$.

(b) If each $\bar{r}_{i}$ is square-free, then the group of automorphism invariants of $A_{\vec{r}}^{(1)}$ is generated by $C_{r}^{c}, \sigma_{\pi}$, and the automorphism invariants $\sigma\left[J_{r}^{m}\right], \sigma[p ; \ell, m], \sigma[\ell, m, n]$, and $\sigma[\ell, m, n, o]$ defined in (i)-(iv) above.

To prove Theorem 3, note that it suffices to fix a prime $p$ and to consider all solutions $a$ to eqs.(13a)-(13c) when the $\bar{r}_{i}$ are all powers of $p$. Both Theorems 3(a),3(b) follow inductively from the following observation. Suppose $\exists \ell$ such that

$$
\frac{\bar{r}_{\ell}}{\operatorname{gcd}\left\{\bar{r}_{\ell}, a_{\ell \ell}\right\}} \geq \frac{\bar{r}_{j}}{g c d\left\{\bar{r}_{j}, a_{j \ell} \bar{r}_{j} / \bar{r}_{\ell}\right\}}, \quad \forall j \text { with } a_{j \ell} \not \equiv 0\left(\bmod \bar{r}_{\ell}\right) .
$$

Then we may take $m_{j}=a_{j \ell} \bar{r}_{j} / \bar{r}_{\ell}: \sigma\left[J_{r}^{m}\right]$ will be an automorphism invariant, and $\sigma_{b} \stackrel{\text { def }}{=} \sigma_{a}$ o $\left(\sigma\left[J_{r}^{m}\right]\right)$ will have $b_{\ell j}=b_{j \ell}=0 \forall j$, by eq.(13e). This means $\left(\sigma_{b} \lambda\right)_{(\ell)}=\lambda_{(\ell)} \forall \lambda \in P_{+}^{r, k}$.

To prove Theorem $3\left(\right.$ a), let $\ell$ satisfy $\bar{r}_{\ell} \geq \bar{r}_{j} \forall j$ with $a_{j \ell} \neq \equiv 0\left(\bmod \bar{r}_{\ell}\right)$. If $\operatorname{gcd}\left\{\bar{r}_{\ell}, a_{\ell \ell}\right\} \neq$ 1 , then replace $\sigma_{a}$ with $\sigma_{a^{\prime}}=\sigma_{a} \circ\left(\sigma\left[J_{r}^{m}\right]\right)$ where $m_{i}=\delta_{i \ell}$ - then eq.(13e) tells us $\operatorname{gcd}\left\{\bar{r}_{\ell}, a_{\ell \ell}^{\prime}\right\}=1$, so eq.(17) is satisfied.

Theorem 3(b) follows from similar arguments. The difficult case there is $p=2$, which is worked out explicitly in [11].

\section{Conclusion}

We begin the paper by reviewing the problem of classifying conformal field theories - in particular what are called their partition functions. An important class of these are the automorphism invariants defined in eqs.(5). This paper classifies all automorphism invariants corresponding to the affinization of $A_{r_{1}} \oplus \cdots \oplus A_{r_{s}}$. This is a necessary and major step toward the full classification of all conformal field theories corresponding to 
those affine algebras. This result, especially the simplicity of its proof, is a strong hint that the full classification should be possible.

We find here that all such automorphism invariants are related in a fairly simple way to the symmetries of the corresponding extended Dynkin diagrams (this will not always be true for other affine algebras $[27,12])$. For $A_{r}^{(1)}$, they all commute and have order 2 (this is not true for most other $g-$ e.g. $\left(A_{r_{1}} \oplus \cdots \oplus A_{r_{s}}\right)^{(1)}$ for $s \geq 2$ ).

A simple sketch of the proof is given at the beginning of Section 2. Our arguments are mostly combinatorial, though a number of algebraic results are required. A remarkable fact used repeatedly is eq.(3a), which says that the ratio of certain entries of the modular matrix $S$ of $g$ equals a certain value of a Weyl character of $\bar{g}$. One place this is exploited is in the rewriting of Verlinde's formula in terms of finite-dimensional tensor product multiplicities.

The arguments here should extend to all other affine algebras [12], but $A_{r}$ is notoriously well-behaved for a Lie algebra so there will be some additional complications, particularly at small levels. The arguments should also lift to more general conformal field theory classifications (namely, what are called the $\mathcal{D}$-type invariants and $\mathcal{E}_{7}$-type exceptionals [11]).

Considering the diverse applications of the representation theory of Kac-Moody algebras, it can perhaps be hoped that the work in this paper - namely the classification of all symmetries of the Kac-Peterson modular matrices - will also find application outside the scope of conformal field theory.

Acknowledgements. I am especially grateful to Philippe Ruelle for convincing me that this problem is solvable, and to Mark Walton for his patient explanations of fusion rules. I also thank Antoine Coste and Jean-Bernard Zuber for general information, and Ron Wang for many helpful stylistic remarks. The hospitality of IHES is also appreciated.

\section{References}

1. Altschuler, D., Lacki, J., Zaugg, Ph.: The affine Weyl group and modular invariant partition functions. Phys. Lett. B205, 281-284 (1988)

2. Bernard, D.: String characters from Kac-Moody automorphisms. Nucl. Phys. B288, 628-648 (1987)

3. Bourbaki, N.: Groupes et Algèbres de Lie, Chapitre IV-VI. Paris: Hermann 1968

4. Bouwknegt, P., Nahm, W.: Realizations of the exceptional modular invariant $A_{1}^{(1)}$ partition functions. Phys. Lett. B184, 359-362 (1987)

5. Cappelli, A., Itzykson, C., Zuber, J.-B.: The A-D-E classification of $A_{1}^{(1)}$ and minimal conformal field theories. Commun. Math. Phys. 113, 1-26 (1987)

6. Coste, A., Gannon, T.: Remarks on Galois symmetry in rational conformal field theory. Phys. Lett. B323, 316-321 (1994)

7. Coste, A., Itzykson, C., Lascoux, J., Ruelle, Ph. (private communications)

8. Fuchs, J.: Simple WZW currents. Commun. Math. Phys. 136, 345-356 (1991)

9. Gannon, T.: WZW commutants, lattices, and level-one partition functions. Nucl. Phys. B396, 708-736 (1993) 
10. Gannon, T.: The classification of affine $\mathrm{SU}(3)$ modular invariant partition functions. Commun. Math. Phys. 161, 233-264 (1994); The classification of SU(3) modular invariants revisited. IHES preprint $\mathrm{P} / 94 / 32$ (hep-th/9404185)

11. Gannon, T.: Towards a classification of $\mathrm{SU}(2) \oplus \cdots \oplus \mathrm{SU}(2)$ modular invariant partition functions. J. Math. Phys. (to appear) (hep-th/9402074)

12. Gannon, T., Ruelle, Ph., Walton, M. A. (in preparation)

13. Gato-Rivera, B., Schellekens, A. N.: Complete classification of simple current automorphisms. Nucl. Phys. B353, 519-537 (1991)

14. Gepner, D., Witten, E.: String theory on group manifolds. Nucl. Phys. B278, 493-549 (1986)

15. Goddard, P., Kent, A., Olive, D.: Unitary representations of the Virasoro and superVirasoro algebras. Commun. Math. Phys. 103, 105-119 (1986)

16. Itzykson, C.: Level one Kac-Moody characters and modular invariance. Nucl. Phys. (Proc. Suppl.) B5, 150-165 (1988)

17. Kac, V. G.: Infinite Dimensional Lie Algebras, 3rd ed. Cambridge: Cambridge University Press 1990

18. Kac, V. G.: talk at Canadian Mathematical Society Meeting on Lie algebras and Lie groups (CRM, Université de Montréal, August 1989), unpublished

19. Kac, V. G., Peterson, D.: Infinite-dimensional Lie algebras, theta functions and modular forms. Adv. in Math. 53, 125-264 (1984)

20. Kac, V. G., Wakimoto, M.: Modular and conformal constraints in respresentation theory of affine algebras. Adv. in Math. 70, 156-236 (1988)

21. Kass, S., Moody, R. V., Patera, J., Slansky, R.: Affine Lie Algebras, Weight Multiplicities, and Branching Rules, Vol. 1. Berkeley, Los Angeles, Oxford: University of California Press 1990

22. Koblitz, N., Rohrlich, D.: Simple factors in the Jacobian of a Fermat curve. Can. J. Math. XXX, 1183-1205 (1978)

23. Ruelle, Ph., Thiran, E., Weyers, J.: Implications of an arithmetic symmetry of the commutant for modular invariants. Nucl. Phys. B402, 693-708 (1993)

24. Ruelle, Ph.: Automorphisms of the affine SU(3) fusion rules. Commun. Math. Phys. 160, 475-492 (1994)

25. Schellekens, A. N., Yankielowicz, S.: Modular invariants from simple currents. An explicit proof. Phys. Lett. B227, 387-391 (1989)

26. Verlinde, E.: Fusion rules and modular transformations in $2 \mathrm{D}$ conformal field theory. Nucl. Phys. B300 [FS22], 360-376 (1988)

27. Verstegen, D.: New exceptional modular invariant partition functions for simple KacMoody algebras. Nucl. Phys. B346, 349-386 (1990)

28. Walton, M. A.: Algorithm for WZW fusion rules: a proof. Phys. Lett. B241, 365-368 (1990)

29. Witten, E.: Non-abelian bosonization in two dimensions. Commun. Math. Phys. 92, 455-472 (1984)

30. J.-B. Zuber, talk given at ICMP, Paris, 1994 